\title{
La innovación en la UNED de Costa Rica. Construcción de una visión sistémica
}

\author{
Innovating at UNED from Costa Rica. A systemic vision construction
}

\author{
Maricruz Corrales Mora ${ }^{1}$ \\ Ana Iveth Rojas Morales ${ }^{2}$ \\ Elisa Delgado Moreira ${ }^{3}$ \\ Jenny Seas Tencio ${ }^{4}$ \\ Marco Sánchez Mora ${ }^{5}$ \\ Universidad Estatal a Distancia, Costa Rica
}

\begin{abstract}
Resumen. Este artículo describe la experiencia de construcción de una visión sistémica para la gestión de la innovación en la Universidad Estatal a Distancia de Costa Rica (UNED). Un grupo de funcionarios, provenientes de diferentes dependencias y Vicerrectorías de la universidad, conformaron un equipo de trabajo e investigación denominado "Nodo de Innovación", en el que se abocaron al estudio de la innovación, que entre otros productos originó la definición de una propuesta para su gestión en la UNED. Con este fin, el grupo ha desarrollado una serie de actividades y procesos en los que se asume a la innovación, como un nuevo eje transversal del desarrollo institucional, con el que la UNED puede manifestarse en el ámbito sociocultural y educativo, a partir de tres formas de expresión posibles, específicas y sostenibles para llevarla a cabo: en lo educativo, lo tecnológico-educativo y lo social. Estos tres tipos de innovación pueden fortalecerse mediante el funcionamiento activo de un sistema y la correspondiente definición y ejecución de una política institucional sobre el tema. El camino recorrido en esta construcción constituye el tema de fondo de este artículo y pretende mostrar la metodología implementada y los productos alcanzados como estrategia global de construcción universitaria en torno a un tema crucial en el contexto actual como es la innovación.
\end{abstract}

Palabras clave. Innovación, sistema de innovación, gestión de la innovación, política institucional.

\begin{abstract}
This paper describes the construction experience of a systemic vision to develop an institutional innovation policy at Universidad Estatal a Distancia de Costa Rica (UNED). A group of employees from different departments and Vicerrectorías of the university, formed a research team called "the Innovation Node", which they devoted themselves to an innovation study, among other products elaborated a definition of one management proposal and system for UNED. For this, the Innovation Node has been developing various activities and processes in order to assume innovation as a central focus of institutional development, in which the UNED can manifest itself in the cultural and educational field from three forms of possible, specific and sustainable innovation to carry out in the educational, technological- educational and social innovation. These three types of innovation can be strengthened by active operation of a system with one associated definition and implementation of a university policy on this issue. These are developing constructions to be approved by university authorities and they are described in this paper. The action taken in this construction is the background issue of this article and aims to show the methodology implemented and the products achieved as a global strategy for university construction around a crucial issue in the current context such as is the innovation.
\end{abstract}

Keywords. Innovation, innovation system, innovation management, institutional policy.

\footnotetext{
${ }^{1}$ Maricruz Corrales Mora, coordinadora Nodo de Innovación. Universidad Estatal a Distancia, Costa Rica. Dirección electrónica: moram@uned.ac.cr ${ }^{2}$ Ana Iveth Rojas Morales, Nodo de Innovación. Universidad Estatal a Distancia, Costa Rica. Dirección electrónica: anrojas@uned.ac.cr ${ }^{3}$ Elisa Delgado Moreira, Nodo de Innovación. Universidad Estatal a Distancia, Costa Rica. Dirección electrónica: edelgado@uned.ac.cr 4Jenny Seas Tencio, Nodo de Innovación. Universidad Estatal a Distancia, Costa Rica. Dirección electrónica: jseas@uned.ac.cr

${ }^{5}$ Marco Sánchez Mora, Nodo de Innovación. Universidad Estatal a Distancia, Costa Rica. Dirección electrónica: msanchezm@uned.ac.cr
} 


\section{Introducción}

El Nodo de Innovación surgió dentro del Proyecto PROY038-2014. Lo aprobó la Vicerrectoría de Investigación como una estrategia metodológica de trabajo y análisis colaborativo con el fin de comprender las concepciones de la innovación en la UNED, la construcción de un referente teórico y el análisis de los requerimientos y estrategias que impulsarán su incremento en el quehacer institucional.

Este camino conduce al planteamiento de la innovación como eje transversal del desarrollo universitario de la UNED. Se tomó en cuenta que es un centro de educación superior a distancia e institución benemérita de la educación y la cultura costarricense, y que se plasme desde la política institucional, de manera que su presencia sea vinculante en todo su quehacer.

El tema de innovación surge a partir de observar que en el ámbito costarricense y hasta regional la UNED es reconocida más allá de su naturaleza universitaria a distancia, que le depara tanto una particularidad distintiva ligada al uso intensivo de tecnologías, como un carácter innovador tecno-educativo, procura mejorar sus procesos de formación superior, en contexto con el ejercicio de una autonomía de libertad académica, promoción del pensamiento crítico y de la consolidación de una ciudadanía activa; retos que generan de por sí procesos de construcción sociales complejos, en los que la innovación puede convertirse en pilar, para la conceptualización y puesta en práctica de cambios específicos. De hecho, debería tomarse como un nuevo lenguaje sobre la realidad y una orientación que estimule lo educativo y social en general.

Cabe señalar que el tema de la innovación no es nuevo en la institución, pues ha sido objeto de análisis en múltiples encuentros académicos y ha sido plasmado en planes y programas, prácticamente desde finales del siglo pasado, cuando como señalan distintos autores, tuvo un importante espacio en las mentes del ciudadano común y de las empresas y/o economías, ya sea porque se le definiera como cambio intencional y controlado, o porque fuera una mejora de algún aspecto concreto de algún sistema, que aportaba un valor agregado (De la Torre, 1998; Drucker, 1998). A esta conclusión, también había llegado el documento de referencia obligada sobre el tema, el Manual de Oslo (OECD, 2005), en el que se afirma como hecho innegable que el desarrollo y la difusión de las nuevas tecnologías (innovaciones) son vitales para el crecimiento económico y la productividad de las naciones.

Más recientemente, algunos autores (Alba, 2009, Ktosemir y Meissner, 2013) amplían esa concepción económica al señalar que la innovación se trata más bien de un cambio basado en conocimiento e ideas que generan valor. Así, el qué o la vía para innovar es el cambio, el cómo o la base es a partir del conocimiento y el por qué o la meta de innovar debe llegar a ser el generar un valor. Desde esa perspectiva, se concibe a la innovación en el corazón del concepto de economía basada en conocimiento.

En este sentido, las universidades por antonomasia son comunidades o redes de conocimientos, cuyo objetivo principal es la creación, acumulación, divulgación y cuestionamiento del conocimiento (David y Foray, 2002). Por tanto, les conviene comprender su gestión institucional, a la luz y acción de las innovaciones.

También, toda innovación conlleva un proceso de construcción social complejo, que por definición asume la existencia de una visión sistémica, caracterizada por componentes o agentes, roles, estrategias y procesos de cambio sostenidos (De la Torre, 1998; Jain, Triandis y Wieck, 2010). Estos cambios pueden ser de carácter específico y tener lugar en cualquier ámbito de la actividad humana, con el propósito de difundirse o consolidarse (Kotter, 2012). De ahí que se trata de 
un nuevo lenguaje sobre la realidad y sus modificaciones, que puede verse como proceso orientado a varias actividades: educativas, productivas, sociales, tecnológicas, científicas y otras. Depende de la misión organizacional para establecer esa orientación con la que se invoque a la innovación, se le promueva y desarrolle. Por eso, en el contexto universitario, implica un enorme reto y complejidad, y un esfuerzo multivariable que demanda en los funcionarios, saber amalgamar en el quehacer institucional con un sentido o vocación innovadora, los procesos, servicios y productos.

La innovación, si se le ve desde el ámbito costarricense, se ha convertido en una política de gobierno, definido por el Ministerio de Ciencia, Tecnología y Telecomunicaciones (MICITT), dado el interés por ver en ella, un motor económico para cambiar las condiciones de vida de los habitantes. Así, uno de los más destacados esfuerzos para asesorar al sector estatal sobre el tema ha sido el Atlas para la Innovación en Costa Rica (MICIT, 2007). En ese Atlas se destacan cinco áreas que obstaculizan los procesos de sistemas de innovación en términos de: a) ausencia de visiones estratégicas y sistémicas; b) limitación en las oportunidades para innovar; c) carencia de sistemas de apoyo financiero; d) deficiente desempeño sistémico de actores y roles, y e) una pobre cultura o limitado capital humano relacionado con el emprendimiento y la innovación.

La UNED no se escapa a los obstáculos citados, muy a su pesar y de las líneas institucionales establecidas en diferentes escenarios y momentos como a) la moción aprobada para favorecer la sistematización de experiencias en innovaciones pedagógicas del II Congreso Universitario (UNED, 2000), b) la descripción puntual de asumir a la innovación como "proceso que permite el mejoramiento continuo del quehacer universitario y sus resultados a partir de la investigación, la generación y aplicación del conocimien- to" (UNED, 2011, p. 41) que se dispuso en el Plan de Desarrollo Institucional 2011-2015 y con la que se pretendía en ese entonces, tomarla como nuevo eje transversal del quehacer institucional.

Aunado a lo anterior, algunas recientes circunstancias, como la elaboración del Plan de Desarrollo Institucional de la UNED del siguiente lustro, el Acuerdo de Mejoramiento Institucional, la actualización del capital humano y los retos propios de la sociedad digital, reafirman la disposición para fundamentar el quehacer de la universidad de la mano de una gestión innovadora. Sin embargo, el planteamiento elegido por el Nodo de Innovación, que se describe en este artículo, no aspira a pensar en la innovación con el énfasis de la corriente económica (OECD, 2005; Mendoza, 2011; Schwab, 2014), que trata más sobre la interrelación academia-gobierno-sector socioproductivo, a partir de la elaboración de productos innovadores, la venta de servicios, la reorganización de la gestión pública y privada y el reconocimiento de ideas como bienes económicos. Más bien, plantea una experiencia de construcción participativa al seno de la universidad, sobre una visión sistémica de la innovación y su quehacer institucional, dada la naturaleza de la organización y su participación en el desarrollo local y nacional.

\section{Metodología}

La metodología seguida por el equipo del Nodo, para la construcción de la visión sistemática de la innovación en la UNED, comprendió tres grandes fases:

I. Construcción de un marco teórico referencial sobre el constructo innovación, tanto a nivel general como en la especificidad del sector educativo universitario público.

En esta fase se dieron las siguientes acciones:

- Revisión y síntesis analítica de la teoría y concepción de la innovación. 
- Revisión de la innovación orientada hacia centros educativos superiores.

II. Exploración de la definición colectiva de la innovación que se posee en la organización desde las personas o grupos innovadores reconocidos como tales.

En esta otra fase se hizo:

-Definición colectiva de la innovación especialmente para y desde la UNED.

- Concurso INSPIRA.

III. Construcción de la propuesta de sistema y de la política institucional de apoyo para el desarrollo de la innovación como eje transversal.

Sus acciones fueron:

- Definición y creación de sistemas, especialmente organizacionales.

- Elaboración de una visión de sistema de innovación universitario.

- Planteamiento de una política institucional de apoyo para el desarrollo del sistema de innovación.

\section{Descripción de las fases}

Fase I. La revisión y síntesis analítica sobre la teoría y concepción de la innovación, tal y como se ha mencionado en la introducción, conlleva a varias dimensiones de análisis, según la literatura, área del conocimiento que se consulte y misión organizacional donde se pretenda desarrollar. Por eso, fue determinante seleccionar y desarrollar una definición propia institucional, sobre lo que implica innovar en general y lo que específicamente podría entenderse en la UNED.

Esto se hizo mediante el estudio y análisis de diversas teorías conceptuales sobre la innovación (clásicos en economía como los de J. A. Schumpeter o en gestión, como los de P. F. Drucker; entre otros), revisión de literatura nacional e internacional sobre su aplicación (como definición conceptual y de algunos sistemas de innovación nacionales, por parte de distintos gobiernos o de empresas que innovan), lo que aportan en la actualidad organismos internacionales que apoyan a la innovación como motor de desarrollo (en mucho señalado por la Organization for Economic Co-operation and Development -OECD), lo que se espera sea el rol protagónico de las universidades en el presente siglo (básicamente lo dispuesto en informes o declaraciones mundiales de UNESCO) y, por supuesto, lo que se habla, comenta y piensa sobre la innovación, a lo interno de la UNED.

Con la revisión de ese tipo de literatura se llegó a una síntesis del concepto innovación, tomando en cuenta la naturaleza específica de la UNED como universidad a distancia e institución benemérita de la educación y cultura costarricense.

Fase II. Otro aspecto importante de esa definición colectiva de la innovación desde la UNED se dio con la definición y la organización del concurso INSPIRA 2014. Un concurso especialmente diseñado por el Nodo de Innovación, para destacar proyectos desde tres ámbitos diferentes (complementarios) de innovación (educativa, tecnológica-educativa y social), que fueron seleccionados por un jurado conformado de especialistas externos y que se vieron beneficiados con una gestión de acompañamiento desde el Nodo para la consecución de los objetivos.

Sin embargo, el conocimiento sobre la opinión de la innovación en la UNED fue obtenido mediante una entrevista.

Fase III. Se efectuó otra revisión de literatura específica, para elaborar una visión de sistema de innovación en la UNED, con el fin de entender qué son los sistemas y cómo se desarrollan como visiones y modelos de trabajo, al seno de las organizaciones. Esto llevó a la mesa de trabajo del grupo, la propia visión de sistema de educación a distancia, y se partió desde esta me- 
todología para pensar en un sistema de innovación, que pudiera ponerse en función, gracias a la estrategia de aprobación de una política institucional sobre el tema.

La elaboración de una visión de sistema de innovación y del planteamiento de una política institucional que le permita desarrollarse llegan a presentar rasgos de síntesis e integración sistémicos, tanto en la forma de conceptualizarlos como en la orientación para implementarlos.

\section{Sujetos participantes}

Diez grupos de funcionarios, cuyos proyectos habían superado el año de trabajo y estaban dentro de campos innovadores, para el contexto de la UNED.

La selección de tales grupos se dio primero en razón de una red institucional de iniciativas diferentes, en el uso de tecnologías digitales, que agrupó al menos a cinco de ellos. Los otros cinco grupos fueron seleccionados por conceso dentro del Nodo de Innovación, a partir de los criterios de selección de que estuvieran trabajando en proyectos, con temas nuevos, en los que hubieran alcanzado un impacto significativo a sus poblaciones meta, según la opinión generalizada dentro de la universidad y se guardara entre los 10 grupos, el equilibrio deseado de disponer casi que igual número de proyectos asociados a cada uno de los tipos de innovación que ya se tenían definidos desde el concurso INSPIRA. Es claro que en la universidad existen más de estos grupos con iniciativas innovadoras. No obstante, con el interés de tener una línea base y un sentido práctico de análisis para iniciar la indagación, estos fueron los seleccionados.

\section{Instrumento para la recolección de información}

Entrevistas a profundidad. El instrumento estuvo conformado por 22 preguntas abiertas, elaboradas por el Nodo de Innovación y organizadas en tres secciones temáticas, a saber: conceptualización (con 8 preguntas), procesos innovadores institucionales (con 9 preguntas) y procesos innovadores del grupo (con 4 preguntas). Al final, se dejó un espacio y tiempo adicional, para comentarios generales y temas emergentes. Así mismo, el cuestionario se estructuró de manera que tuviera: a) precisión, sobre la visión conceptual de la innovación que los funcionarios tienen interiorizada y pueden expresar; b) amplitud descriptiva, de las prácticas grupales innovadoras que han venido realizando y c) aentido crítico, para describir o proponer los cambios que la institución demanda, en razón de su mejoramiento y los procesos innovadores.

Las entrevistas se planearon con la presencia y participación de todos los miembros del grupo, ya que interesaba recoger las respuestas, de manera grupal y espontánea por parte de cada uno de ellos. La aplicación de las entrevistas demandó una dedicación prácticamente de medio año debido a la metodología de la aplicación y la búsqueda del mayor número de miembros de los grupos innovadores. Se empezó a finales del año 2014 y se concluyó a inicio del 2015.

Cada entrevista tomó alrededor de dos horas (una validación inicial hecha por el Nodo, respondiendo el cuestionario de la entrevista, dictó ese tiempo calculado); se le pasó a revisión filológica y también se remitió a los miembros del Jurado externo que tuvo el concurso INSPIRA, para tener sus impresiones y observaciones.

En cada sesión de entrevista, la coordinadora del Nodo de Innovación se hizo acompañar de al menos un miembro del Nodo, se solicitó consentimiento de grabación auditiva al grupo entrevistado y, se levantó la transcripción mediante digitación, que en cuestión de días se les mandó al grupo, con el fin de que enviaran su aval escrito.

Técnicas de análisis. Con la información recolectada, el Nodo de Innovación procedió a realizar un análisis cualitativo que luego divulgó por 
diferentes medios en la universidad. Primero, se usó la aplicación digital Atlas.ti, en la que se definieron códigos de análisis cualitativo, para luego conformar categorías analíticas, desde los análisis interpretativos previos realizados por los miembros del Nodo de Innovación.

Análisis y sintesis. Luego de una revisión de literatura referente a la innovación y su enfoque en organizaciones distintas (De La Torre, 1998; Drucker, 1998; OECD, 2005; Altback et al., 2009; Bermúdez et al., 2009; Dron, 2014), el Nodo de Innovación asume como propia y, especialmente dado el contexto universitario de la UNED, la definición de Alba (2009); autor que si bien es del área empresarial, propone una visión sistémica importante sobre la innovación, que a la letra dice así: "la innovación es todo cambio basado en conocimiento que genera valor" (p. 43).

Con esa definición, se sobrentiende el qué o la vía (refiriéndose al cambio que provoca la innovación), el cómo o la base (mediante el conocimiento) y el porqué o la meta (el hecho de transcender o generar algún valor). De ahí la importancia de asumirla, a nivel institucional, porque así la innovación se concibe como proceso, que se deriva del conocimiento y que está orientada hacia metas específicas.

Un proceso es el desarrollo de una acción continua, en una dirección predeterminada y con un propósito definido, que no responde solo a una situación aislada ni a una serie de tareas relacionadas, porque todo proceso tiene una finalidad y contribución que debe aportar al sistema donde reside (López, 2011).

Además, con esa definición de innovación, se coincide plenamente con la especificada en la UNED (junio 2011), en el Plan de Desarrollo Institucional 2011-2015: “...proceso que permite el mejoramiento continuo del quehacer universitario y sus resultados a partir de la investigación, la generación y aplicación del conocimiento" (p.
41); tanto por la concepción de proceso, como por el aspecto de mediar el conocimiento.

También, sirve como una definición complementaria, de lo que expresaron recientemente los grupos innovadores entrevistados por el Nodo de Innovación: la innovación se determina por el contexto en donde se desarrolla o aplica, es relativa a los recursos existentes, sirve para mejorar procesos que deben ser continuos o constantes, e impacta positivamente a la organización transformando su quehacer (Corrales et al., 2016).

A partir de lo anterior y con el fin de delimitar los posibles tipos de innovación a los que puede abocarse la UNED, se hizo necesario especificar: qué la caracteriza del resto de instituciones educativas y qué determina su esencia.

Al respecto, el Nodo de Innovación sugiere que la UNED fortalece su identidad, por medio de: a)La condición y naturaleza de institución de educación superior (La Gaceta, 1977), b)La definición de que es una universidad a distancia desde su creación (La Gaceta, 1977), y c)La designación de institución benemérita de la educación y cultura costarricenses (La Gaceta, 2007).

$\mathrm{Al}$ tomar en cuenta esas condiciones socio-históricas de la UNED, por las que responde la misión institucional, se pueden definir tipos específicos de innovación, con los que la institución puede consolidar y desarrollar procesos, servicios y productos relacionados. Estos tipos serían: innovaciones educativas, innovaciones tecnológico-educativas e innovaciones sociales (respectivamente de acuerdo con esos tres señalamientos) y pueden presentarse en varias combinaciones.

Por innovación educativa se concibe el mejoramiento, la transformación y el progreso de procesos vinculados con el desarrollo cognitivo-social de los individuos y de la práctica profesional educativa. Sería, entonces, el motor para mejorar rendimientos a escala institucional, 
apropiarse de principios organizadores y éticos que permiten vincular los saberes, dar sentido a una vida plena humana y a un nuevo espíritu científico, desde los cambios o renovaciones de las prácticas educativas a distancia, en áreas como la curricular, la didáctica, la evaluación de los aprendizajes, la formación en general, la cognición y el aprendizaje, entre otros.

Por innovación tecnológico-educativa se entiende la apropiación pertinente o la producción de distintas tecnologías, que pueden adaptarse o crearse como recurso y forma de mediación en los procesos educativos a distancia que disponga la universidad. Cada vez son más complejas las formas de aprender gracias a la integración tecnológica en los ambientes educativos, sobre todo, a la manera en que la tecnología viene reorganizando el modo en que la gente vive, se comunica y aprende en general, que como lo dicta el Conectivismo (Siemens, 2005), ahora se hace de manera colectiva, dado que, cada vez, gusta más aprender mediante las conexiones socioculturales y en red.

Por innovación social se espera dar los cambios significativos en las relaciones sociales de comunidades, regiones o sociedad, a través de procesos de movilización-participación y acciones en los que la UNED propicie mejoras en las relaciones sociales, de estructura de gobierno o de mayor empoderamiento colectivo, mediante investigaciones multi y transdisciplinarias $y$, de modo que crezca un tipo emergente de comportamiento social, con el que se defiendan los valores de solidaridad, equidad, inclusión y bienestar (una explicación adicional sobre estos tres tipos de innovaciones, con los que la UNED puede identificarse, están descritos en el artículo de Corrales, 2014).

La construcción colectiva sobre la conceptualización innovadora empezó a darse, primero, mediante las entrevistas, al conocer la opinión de los grupos de funcionarios identificados y se- leccionados. La información recolectada visibilizó la noción de innovación, lo que no es, cómo se le aprecia al seno de la universidad, cuáles son sus condiciones y limitaciones y por qué podría ser necesaria una política institucional, entre otros tantos aspectos tratados en ese proceso indagatorio.

Una segunda forma se dio a través de la creación del concurso INSPIRA, que se gestó desde el Nodo de Innovación con el apoyo de la Vicerrectoría de Investigación, para promover la investigación científica bajo los tres tipos anteriores de innovación definidos (un proyecto premiado para cada tipo). La experiencia de acompañamiento del Nodo de Investigación para los investigadores de los tres proyectos premiados, resulta tener una excelente acogida por parte de ellos; tanto por sentirse apoyados en el cumplimiento de los objetivos respectivos de los proyectos, como en la facilitación de los procesos y posterior divulgación que sobre ellos se hizo durante la presentación institucional del Nodo de Innovación y su quehacer. INSPIRA fue visto como un fuerte impulso inicial, para definir una política necesaria en la UNED, con la que se pueden fortalecer las iniciativas innovadoras.

De esta manera, también puede verse que el Nodo de Innovación tuvo acceso a experiencias innovadoras que ya tenían tiempo de estarse dando, así como de aquellas, que por el concurso, apenas iniciaban y se les podía dar seguimiento desde el inicio de su labor. Ambas formas permitieron conocer, de primera mano, las diferentes vías de gestión de los procesos innovadores en la UNED.

Acerca de lo anterior, Johnson (2001) explica que si se toma a la innovación como una función esencial de la organización, esta requiere de incentivos y recursos, guiar la dirección en las búsquedas oportunas y pertinentes, reconocer el potencial para innovar y facilitar el intercambio de la información y el conocimiento; es decir, la 
institución debe asumirla dentro de cada uno de sus procesos claves, tanto como universidad a distancia como por institución benemérita y esto da cabida al diseño y desarrollo de un sistema de innovación para la UNED.

Los elementos de un sistema deben guardar coherencia entre sí (como principio básico); de lo contrario, surgen muchos problemas personales, grupales, organizacionales y hasta de extensión mayor.

Una lista de elementos propios de un sistema por considerar, en el diseño del sistema de innovación para la UNED, es la siguiente:

-propósito (significado y razón de ser del sistema), lida),

-recursos (de entrada, procesamiento y de sa-

-información (que definen el estado del sistema en un tiempo dado),

-procesos (procedimientos y/o actividades o "rutinas" que mantienen la estabilidad o variación del sistema),

-medición o evaluación (elementos que facilitan la tarea comparativa entre el estado actual y deseable),

-productos o resultados (lo que produce como consecuencia de los procesos),

-límites (dimensiones y magnitud del sistema para identificarse y diferenciarse en el medio) y

-realimentación (proceso que facilita la autorregulación y funcionamiento del sistema según su propósito) (López, 2011).

Siguiendo la lista, el sistema de innovación de la UNED llega a plantearse por las innovaciones que agentes o actores (funcionarios, estudiantes, instancias, grupos y comunidades vinculadas con la institución) producen, de forma continua, para el desarrollo del modelo educativo a distancia y de sus objetivos institucionales, mediante procesos, productos y/o servicios, mejoras en la organización, en el desarrollo y capacidad del talento humano institucional y en la forja de vínculos institucionales y procesos de interna- cionalización que son finalmente las funciones intrínsecas o vías con que generalmente se promueve la innovación en una organización (Alba, 2009). Para el caso de la UNED y de acuerdo con los Lineamientos de Política Institucional 20152019 (UNED, 2015). Esto no responderá a solo un modelo, sino a una amplia gama de métodos, técnicas y tecnologías que se aprovecharán con flexibilidad (p. 26).

De lo anterior, las definiciones conceptuales de los componentes sistémicos presentados se establecen de la siguiente manera para el contexto de la UNED:

- El propósito del sistema será la generación de las innovaciones en el cumplimiento de los fines esenciales de universidad a distancia y como institución benemérita de la educación y cultura costarricenses.

- Una innovación será la acción de un grupo de profesionales (de diferentes áreas del conocimiento, preferiblemente de diversas instancias), estudiantes o miembros de comunidades, que pueden conformar redes de trabajo y/o conocimiento, con una dirección predeterminada y propósito definido, y se expresan institucionalmente desde las categorías de la innovación educativa, la innovación tecnológica-educativa o la innovación social. Es así como de ahora en adelante, una innovación, desde la visión de este sistema, se concebirá dentro de los procesos institucionales, con los que se sostendrá el propio sistema de innovación y brindarán así productos o servicios.

- Los actores de este sistema son tanto las instancias funcionales de la universidad como también aquellos grupos (en comisión, grupos temáticos de acción investigativa o de extensión universitaria, comunidades sociales o redes universitarias), que se organicen por un interés común y sean capaces de orientarse para generar una innovación. 
- Cuando un proceso es relevante, se observa con facilidad la acción que realiza, cómo integra varias tareas y actividades con un esfuerzo de coordinación y optimiza recursos conducentes a una mayor efectividad. Por eso, los tres tipos de innovación, sugeridos para la UNED, deberán llegar a ser procesos relevantes en la consecución de la misión institucional.

Finalmente, las organizaciones son sistemas adaptativos complejos que consisten de muchos agentes (gente y grupos que dentro de un espacio limitado trabajan bajo situaciones delimitadas organizacionalmente y de forma interdependiente). Son complejos en términos de interacciones, reglas y convenciones socioculturales y se adaptan según patrones de comportamiento que derivan de esas reglas y de convenciones de autorganización, para mutar en el tiempo y dar respuesta a circunstancias que ocurren en el curso de las interacciones (Smith, 2015).

Por su parte, las políticas son decisiones orientadoras que influyen en los procesos que desarrolla un organismo social o área determinada, en forma coherente y consistente. Tienen que ver con una visión de mundo total o parcial o con alguna doctrina, ideología o filosofía y, forman un cuerpo para el vínculo de sus miembros. En el ámbito de las universidades y especialmente de las públicas (como es el caso de la UNED), las políticas universitarias derivan de una visión clara de la naturaleza institucional (D'Alton, 2011).

Aunado a lo anterior, con lo aprobado en los Lineamientos de Política Institucional 2015-2019 (UNED, 2015), que a la letra dice así:

El modelo de gestión de la universidad debe elaborarse específicamente en función de la academia y con el fin de fortalecer su calidad: debe fundamentarse en enfoques y conceptos apropiados, pertinentes y actualizados de las ciencias sociales y la administración, en perspectivas científicas y filosóficas pertinentes para comprender las organizaciones humanas y en las concepciones éticas y filosóficas fundamentales de la universidad y de su papel en nuestra sociedad, según ello ha quedado formulado en los documentos fundadores de la UNED, los acuerdos de los congresos universitarios y en estos Lineamientos (L107, pág. 77).

La UNED debe analizar su estructura y funcionamiento internos, especialmente en cuanto a la articulación e interrelación de las entidades que la integran, con miras a posibles reajustes o a una reorganización sistémica. Es esencial fortalecer la cooperación y coordinación entre el ámbito académico y el administrativo, en la comprensión de que ambos desempeñan un papel esencial para el logro de los objetivos de mejoramiento académico que estos Lineamientos definen (L111, págs. 78 y 79).

Se llega a la necesidad de definir y aprobar una política institucional que dé vida a ese sistema de innovación, en la que se le aprecie (al sistema de innovación de la UNED) como un modelo o representación gráfica de la realidad, donde se reconozcan a los actores (instancias, grupos o redes), lo que estarían haciendo de innovador (productos y servicios), de qué manera (procesos) y con cuáles recursos (gestión del capital humano, vínculos y forma organizacional).

Este planteamiento llega a verse viable solo si se integran las visiones sistémicas de la educación a distancia con la nueva visión sistémica de la innovación; es decir, al considerar, primero, a las áreas funcionales de la universidad (docencia a distancia, investigación, extensión y gestión universitaria) como funciones esenciales (o macro-procesos institucionales) y, a sus procesos, lo que reúne a una o más dependencias, grupos o redes de la UNED (actores del sistema), en el cumplimiento de las funciones y de la generación de productos/servicios. Los procesos relacionados, que involucran a una o más entidades, dan lugar a lo que puede llamarse una función esencial (o área fundamental desde el sistema 
de educación a distancia), que deben ejecutarse en el cumplimiento directo o indirecto de uno o más objetivos de la organización.

Una vez que se piensa en esa fusión de sistemas (el de educación a distancia y el de innovación), hay que prever cómo se generarán las innovaciones en la institución desde los diferentes actores, instancias, grupos o redes. Específicamente por:

a)los productos y servicios que brinda la UNED,

b)la gestión por procesos que se realiza para el logro de sus objetivos,

c)el capital humano y su respectiva forma organizacional, que puede facilitar o no las innovaciones $\mathrm{y}$

d)el aporte novedoso que con su práctica educativa y cultural puede ofrecer a favor del mejoramiento y desarrollo de la educación a distancia, a través de vínculos y procesos de internacionalización.

Tales formas de innovar (actos o funciones del sistema de innovación dentro de la gestión a distancia) propician sinergia en ambos sistemas, uno como base del otro, que visualmente pueden representarse mediante hélices que dinamizan el ejercicio académico de la UNED, y a la vez, pueden llegar desde fuera a la institución para transformarla. La figura 1 es un primer intento gráfico de representar esta propuesta sistémica.

La política institucional se requiere para disponer de una clara definición de la innovación en la UNED, con la que se guíe la academia y gestión universitaria, en la realización de innovaciones educativas, innovaciones tecnológica-educativas e innovaciones sociales, capaces de seguir transformando el quehacer institucional y la educación a distancia ejercida por la institución. De hecho, cuatro enunciados definen a la política en su conjunto, con estrategias de acción asociadas para su ejecución.

\section{Reflexiones y aportes finales}

El Nodo de Innovación considera que, esta nueva visión sistémica de la innovación apoyada en una política institucional demanda identificar,

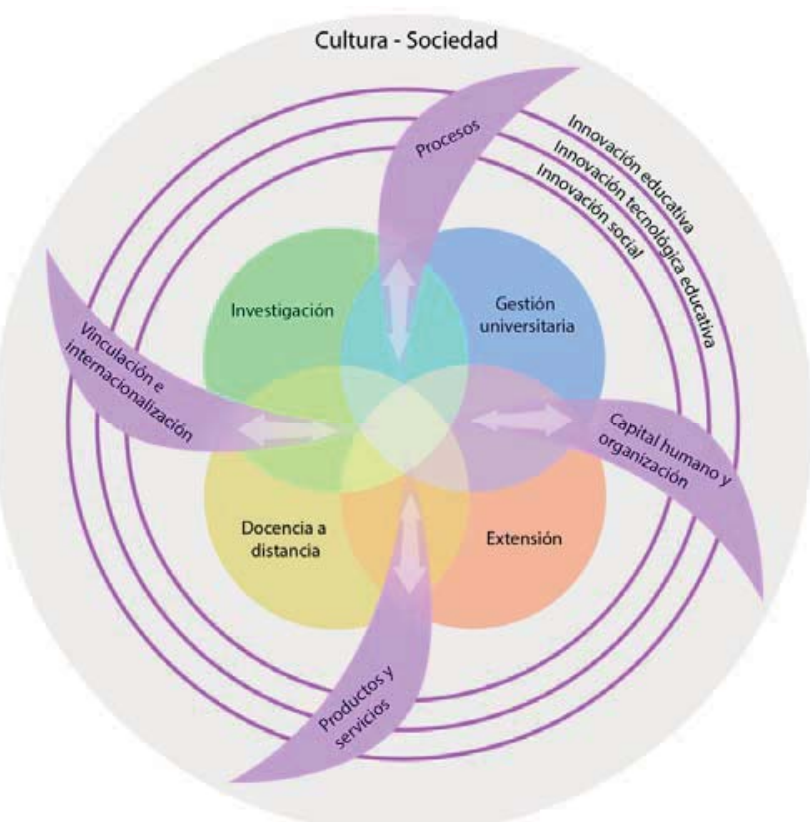

Figura 1. Representación sistémica de la innovación en la UNED a partir de su condición de universidad a distancia. 
por consenso a lo interno de la universidad, los procesos más importantes o propicios de innovar en cada área fundamental o macro-proceso, saber cuáles dependencias los generan mediante los respectivos procedimientos, cuáles productos o servicios brindan, a partir de cuáles capacidades de la organización y de su capital humano y con cuáles vínculos externos asociados. Lo anterior implica realizar un mapeo funcional y estructural de la institución, que valore las interrelaciones más relevantes, si existen interdependencias funcionales, duplicidades de esfuerzos (o actividades procedimentales), todo ello para que permita al final de ese levantamiento informativo de la gestión institucional, mejorar las bases del sistema de educación a distancia de la UNED, con la sinergia complementaria del sistema de innovación.

Por supuesto, es necesario contar con el apoyo de las instancias técnicas de la Vicerrectoría de Planificación, en coordinación con lo planteado para el análisis organizacional, de manera que si se quiere aprobar la política institucional planteada sea porque la innovación forma parte intrínseca y se considere eje transversal del desarrollo institucional definido en el siguiente Plan de Desarrollo Institucional.

Conlleva, además, una intención clara de participación de las diferentes dependencias y áreas del conocimiento, para seguir integrando los procesos claves funcionales de la universidad y mejorarlos desde esta visión sistémica. Se recomienda que una vez que se disponga del análisis funcional administrativo por procesos o áreas claves, se validen mediante talleres o consultas a las dependencias y se verifiquen las interdependencias que la organización tiene, en aras de simplificar y alcanzar más efectivos procesos y funciones.

Tanto la propuesta, como la conformación del Nodo de Innovación, puede decirse que exploran dentro de la UNED, la actualización de la gestión universitaria, porque más allá de la fuerza con la que se impone una estructura organizativa, el planteamiento de tal visión sistémica se ha alcanzado gracias al aporte de diferentes profesionales, de áreas del conocimiento diverso, sobre todo, de dependencias de distintas Vicerrectorías.

El tema de la innovación enriquece tanto a nivel individual, como al grupal y organizacional. Los tiempos actuales demandan asumir los nuevos retos de las sociedades con recursos novedosos y la superación de nuestras individualidades. Este podría ser un nuevo modelo de gestión universitaria a distancia o cultura institucional, que expuesto ahora mediante este artículo y actividades de divulgación por parte del Nodo de Innovación, ya no depende solo de este, sino de la aprobación respectiva de las autoridades universitarias y de la obligada interiorización, especialmente por parte de los funcionarios, para hacerla parte de su realidad y quehacer.

\section{Referencias}

Alba, M. (2009). I-empresarios. Madrid: LID Editorial Empresarial.

Altbach, P. G.; Reisberg, L. y Rumbley, L. E. (2009). Tras la pista de una revolución académica: Informe sobre las tendencias actuales. Resumen. Conferencia Mundial sobre la Educación Superior, organizada por la Unesco, Francia. Recuperado en http://unesdoc. unesco.org/images/0018/001831/183168s. pdf

Bermúdez, J. C.; López, S.; Carrillo, M. A.; Segura, G.; Agüero, D.; Vargas, M.; Aguilar, G.; Chinchilla, A. y Ramírez, H. (2009). Dinámicas de la innovación en las instancias del sector público de nivel político del Sistema Nacional de Ciencia y Tecnología de Costa Rica. Heredia, Costa Rica: Escuela de Relaciones Internacionales, Universidad Nacional. 
Corrales, M. (Diciembre 2014). Innovación universitaria en la UNED. Revista Innovaciones Educativas, (16)21, pp. 73-83, San José: Universidad Estatal a Distancia.

Corrales, M.; Delgado, E.; Rojas, A. I.; Seas, J.; Sánchez, M.; Sevilla, G. (Octubre, 2016). La innovación en la UNED: Bases de su conocimiento. [En espera de publicación].

D'Alton, C. (2011). Las políticas académicas en la UNED. En IV Congreso Universitario de la UNED. Innovaciones en la gestión académica del modelo educativo de la UNED. San José, Costa Rica. Recuperado en http://www. uned.ac.cr/ivcongreso/conferencias/conf02. shtml

David, P. A. y Foray, D. (Marzo del 2002). Una introducción a la economía y a la sociedad del saber. Revista Internacional de Ciencias Sociales, No. 171. Paris, Francia: UNESCO. Recuperado en http://www.unesco.org/ new/fileadmin/MULTIMEDIA/HQ/SHS/ pdf/171-fulltext171spa.pdf

De la Torre, S. (1998). Cómo innovar en los centros educativos. Estudio de casos. Barcelona, España: Escuela Española.

Dron, J. (2014). Innovation and change: Changing how we change. En Zawachi-Richter, O. y Anderson, T. (2014) (Eds.) Online Distance Education. Towards a research agenda, pp. 237-265, Athabasca, Canada: AU Press.

Drucker, P. F. (Noviembre-Diciembre, 1998). The discipline of innovation. En Harvard Business Review, pp. 3-8, MA, EE.UU: Harvard reprint 98604.

Jain, R. K.; Triandis, H. C. y Weick, C. W. (2010). Managing research, development and innovation. Managing the unmanageable. 3rd edition. New Jersey, EE.UU.: Wiley \& Sons.

Kotsemir, M. y Meissner, D. (2013). Conceptualizing the innovation process - Trends and outlook. Basic research program. Working papers. Series: Science, technology and innovation WP BRP 10/STI/2013. National Research University Higher School of Economics. Russia.

Kotter, J. P. (2012). Leading change. Boston, EE.UU.: Harvard Business Review Press.

Johnson, A. (2001). Functions in innovation system approaches. [Ponencia]. En Nelson and Winter Conference, realizada del 12 al 15 de junio del 2001. Aalborg, Suecia. Recuperada en http://www.druid.dk/conferences/ nw/paper1/a_johnson.pdf

La Gaceta (2007, 22 de noviembre). Universidad Estatal a Distancia, declarada Benemérita de la Educación y Cultura de Costa Rica. Decreto de la Asamblea Legislativa No. 8623. San José, Costa Rica: Imprenta Nacional.

------. (1977, 12 de marzo). Ley de creación de la Universidad Estatal a Distancia. Decreto de la Asamblea Legislativa N. ${ }^{\circ}$ 6044. San José, Costa Rica: Imprenta Nacional.

López, R. (2011). Inteligencia sistémica. Fundamental para el éxito. México: Trillas.

Mendoza, P. (2011). El capitalismo académico en el cuadrante de Pasteur. University of Florida, EE.UU. Recuperado en http://education.ufl. edu/mendoza/files/2011/02/JFHE-v33n3.pdf

Ministerio de Ciencia y Tecnología - MICIT (2007). Atlas para la innovación en Costa Rica. Informe final. San José, Costa Rica: Editorama S. A.

Organization for Economic Co-operation and Development - OECD (2005). The measurement of scientific and technological activities. Proposed guidelines for collecting and interpreting technological innovation data. Oslo Manual. Eurostat Commission. 
Schwab, K. (2014) (Ed.). The global competitiveness report 2014-2015. Insight report. Suiza: World Economic Forum.

Siemens, G. (January, 2005). Connectivism: A learning theory for the digital age. International Journal of Instructional Technology and Distance Learning. (2)1 Recuperado en http:// www.itdl.org/journal/jan_05/index.htm

Smith, R. (2015). A change management perspective. En R. Smith, D. King, R. Sidhu y D. Skelsey (Eds.), The Effective Change Manager's Handbook. Essential guidance to the change management body of knowledge, pp. 1-77, London, UK: The APM Group Limited.
Universidad Estatal a Distancia - UNED (20 de febrero del 2015). Lineamientos de política institucional 2015-2019. Aprobados en sesión 094-2015, Art. I, celebrada el 2 de febrero del 2015, en la Asamblea Universitaria de la UNED. San José, Costa Rica.

-------. (Junio 2011). Plan de Desarrollo Institucional 2011-2015: Para el fortalecimiento de la educación a distancia. Aprobado por el Consejo Universitario, sesión 2101-2011. San José, Costa Rica: Vicerrectoría de Planificación de la UNED. 